\title{
Heavy Truck Drag Reduction Obtained from Devices Installed on the Trailer
}

\author{
Luigi Salati, Federico Cheli, and Paolo Schito \\ Department of Mechanichal Engineering, Politecnico di Milano
}

\begin{abstract}
The flow-field around a "common" European heavy truck, equipped with several different trailer devices, is investigated using steady and unsteady simulations.

This work demonstrates how with simple devices added on the trailer it is possible to strongly decrease the aerodynamic drag over $10 \%$, with an increase of overall dimensions below $1 \%$ without any change to the load capacity of the trailer.

Several devices, installed on the trailer, are tested on a target vehicle and the shape of the "airbag", the "fin", the "boat tail" and the "front-rear trailer device" has been optimized to achieve the maximum in drag reduction in front wind. The performance of the optimized devices are tested also in cross wind conditions with the yaw angle varying from $0^{\circ}$ to $30^{\circ}$.

The truck equipped with the front-rear trailer device is also investigated using time variant simulation with yaw angle of $0^{\circ}, 5^{\circ}, 10^{\circ}$.

Detached eddy simulation (DES) with one equation Spalart-Allmaras as turbulence model is used to perform the analysis of the unsteady flow; the two equation k- $\omega$ SST turbulence model is selected for the steady-state RANS simulations. The unsteady simulations are performed using computational meshes on the order of 40 million of elements while RANS simulation are done with mesh of 10 million of elements.
\end{abstract}

The analysis is performed in the open-source CFD framework OpenFOAM ${ }^{\circledR}$.

\section{INTRODUCTION}

The research effort in heavy truck industry in recent years is focused on fuel efficiency.

The main motivations are:

- the regulations about emissions are increasingly more restrictive and require a continuous development on the final product;

- the rise of the fuel price;

- the optimization of the operational costs of the trucks.

It is estimated that $50 \%$ of the truck engine power is needed to overcome aerodynamic drag at typical highway speed of 90-100 $\mathrm{km} / \mathrm{h}$. In fact aerodynamic drag increases to the square of the vehicle speed, and is the main source of fuel consumption at cruise speed (Schoon [1] ). It is estimated that a reduction of $20 \%$ in the drag coefficient results in 10\% fuel efficiency increase (National Research Council Canada [ㄹ]).
A crucial point, for aerodynamic efficiency is the regulation concerning the maximum dimension for the truck-trailer. This is true especially in Europe (Directive 96/53/EC []]), where the maximum permitted length for a heavy truck is measured considering the total length of the vehicle (tractor + trailer), while in the US the regulation prescribes only the maximum length of the trailer as shown in the Figure 1. This regulation influences strongly also the tractor shape and its aerodynamic performance.

Many studies have been performed in the past decades both in wind tunnel and road test to investigate the flow-field around the trucktrailer. In the last years Computational Fluid Dynamics (CFD) studies are becoming more common due to the increase of High Performance Computing (HPC) performances and the computational power available for the simulation. The complexity of the geometry of the vehicle and the strongly-transient behavior of the flow-field require very detailed computational meshes: more than 20 million nodes are necessary to run a transient simulation with really small time step.

This is the accepted version of Salati L., Cheli F., Schito P., Heavy Truck Drag Reduction Obtained from Devices Installed on the Trailer (SAE Int. J. Commer. Veh., $2015,8: 2,747-780)$.

The final publication is available at: http://dx.doi.org/10.4271\%2F2015-01-2898 


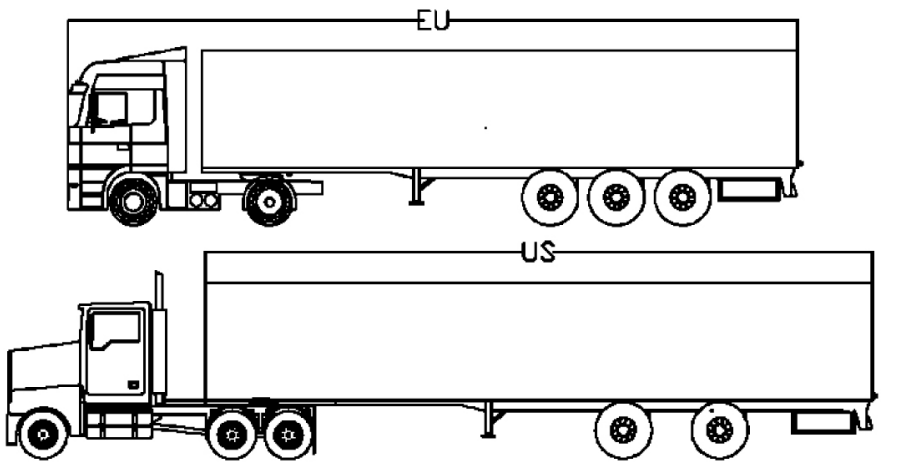

Figure 1. Comparison between European and American regulation regarding heavy truck maximum dimension.

The main areas that influence the overall drag coefficient of the heavy truck are:

- $\quad$ the front corner radius of the tractor (Palowski [4] $)$;

- $\quad$ the tractor-trailer gap (Hucho [ [ $]$ );

- $\quad$ the underbody of both trailer and tractor (Buchheim et al.[్] and Howell [7]);

- the interaction between the tractor and the trailer (Gilhaus[ㅈ]).

A wide range of devices can be developed for drag reduction on the tractor while for the trailer less solutions are practically used in the industry due to the limits of the current regulation and for technical issues (for example the access to crucial area of the trailer for maintenance, etc.).

Also economic reasons influence the current "box" shape of the trailer: box shaped trailers have higher load capacity compared to stream-line shape, easy access to the merchandise, easy delivery as a container on shipping boat, street dimension (height of the tunnel), etc. Another crucial aspect to take into account is the safety requirement of these devices: they must not increase the risk of injury for the passengers in case of crash with others vehicles.

The most common drag reducing devices are:

- $\quad$ cab roof fairings and side fairings (9-17\% drag reduction Drollinger [9]; Cooper [10]; Leuschen and Cooper [11]);

- $\quad$ trailer-front fairing (7-10\% drag reduction - Garry [12]; Watkinset al. [13]);

- $\quad$ tractor and trailer side-skirts (4-6\% drag reduction - Garry [14]; Ingram [15]);

- boat-tail and base flaps (7.5\% drag reduction - McCallen et al. [16]; Cooper [1ㅇ]).

In recent years some studies have been performed on active drag reduction devices, such as boundary layer suction, but their complexity and the necessity to provide energy for their operation did not allowed to these devices to reach the market.
Another interesting way to reduce drag for the heavy truck transport is platooning as shown in Schito [17]. This solution requires a significant change in the infrastructure and in the driving behavior of the vehicle surrounding the platoon. For all these reasons commercial platooning is currently not available.

One of the main issues in the truck aerodynamics, is the clearance gap that has to be provided between tractor and trailer. If the gap is very small, the turning capacity of the vehicle will be strongly affected, while, with a large gap, the vehicle tends to behave as two separate bodies that generate twice the drag of a single body. This effect is amplified in cross-wind when the vehicle is yawed relative to the wind direction (Gilhaus []]).

In this paper external devices that can be applied to the existing truck shape will be studied and developed in order to increase the aerodynamic efficiency of the truck, decreasing the fuel consumption of the vehicle with both economic and environmental benefit. The idea is to keep fixed the internal load capacity of the trailer and no radical changes on the truck body shape will be introduced: the proposed devices will be fixed to the existing trailer geometries. External devices can be easily applied to existing trucks with limited cost and high benefit in terms of fuels saving and installation costs. It must also be noticed that in this way no significant change must be done to logistic centers or shipping boats or in general to the whole commercial transport system. No modifications are proposed on the tractor since the modifications may influence also other features such as cooling, combustion efficiency and engine area arrangement.

The analysis will be conducted using open-source CFD software as OpenFOAM ${ }^{\circledR}$ and Paraview ${ }^{\circledR}[\underline{19}]$, analyzing the flow around the truck using state-of-the-art solvers and methods.

\section{TARGET VEHICLE}

It is decided to use a truck geometry corresponding to a common truck currently available on the EU streets. The heavy-truck model is simplified for CFD studies (see Figure 2): the cooling system and bracket that holds the mirrors are neglected, as well as the turning lights and the vehicle registration plate holder.
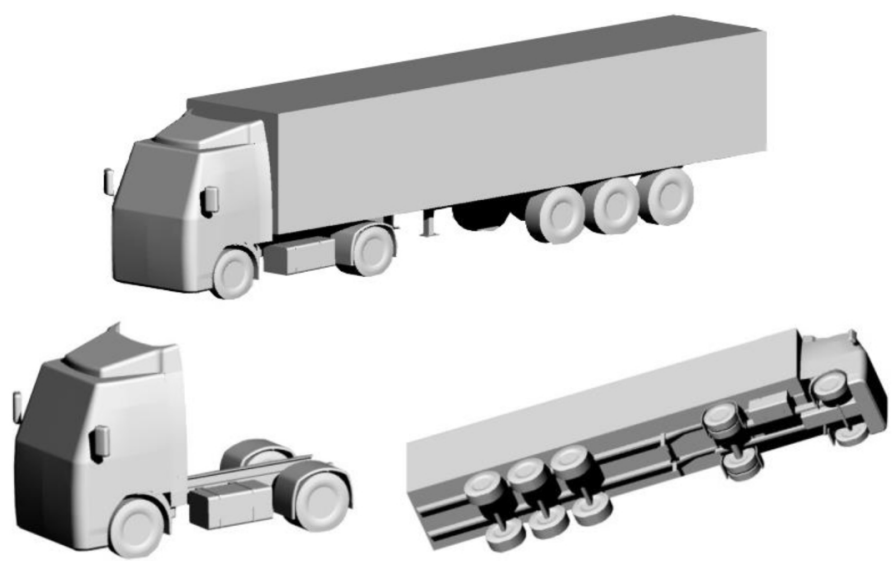

Figure 2. Heavy truck geometry simplified geometry used as target vehicle. 
The base model is already provided with cab roof fairings, side fairings, wheel houses for the tractor tires and a suitable design of the front corner radius of the tractor. All these devices are currently available on most European heavy truck.

The lower part of the wheels are opportunely modelled to reproduce the contact area between the tires and the ground and the deformation of the wheels. The target vehicle overall dimensions (without the mirrors) are reported in Table 1.

Table 1. Truck overall dimensions.

\begin{tabular}{|c|c|c|}
\hline lenght & width & height \\
\hline$[\mathrm{m}]$ & {$[\mathrm{m}]$} & {$[\mathrm{m}]$} \\
\hline 15.8 & 2.5 & 3.9 \\
\hline
\end{tabular}

\section{EVALUATED DEVICES}

Taking into account only the aerodynamic features of the trailer, the main contribution to drag are: the rear of the trailer due to the vortices detaching from the top, the bottom and the sides of the rear faces of the trailer, and the underbody. This consideration leads to design aerodynamic devices especially influencing these areas. Different shapes are tested: "Airbag" (see Figure 3-a), "Fin" (see Figure 3-b), "Boat tail" (see Figure 3-c) and "Front trailer device" (see Figure $\underline{3-\mathrm{d})}$. All the geometries are tested in both front and cross-wind.


(a)

(b)

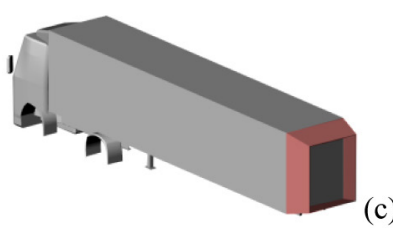

(d)

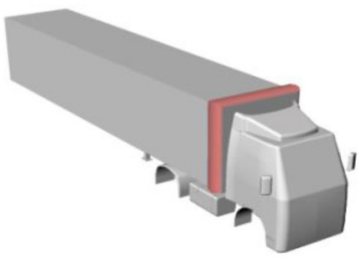

Figure 3. Devices installed on the trailer: Airbag geometry (a), fin geometry (b), boat tail (c), front trailer device (d).

The "Airbag" (Figure 3-a) is designed to decrease the impact on the drag of the vortices generated in the rear of the model, mainly from the top and the bottom of the trailer. It will work also as an airbag in case of crash when another vehicle is approaching one with this device installed in the rear part of it, if the structural design takes into account possible shock absorbing properties. A proper design for this device allows to access the trailer load area, but some issues may arise when placed on a cargo vehicle or in a cargo handling area since the space occupied by this device may interfere with the cargo infrastructure standards.

The "Fin" device (Figure 3-b) is modelled to influence the vortices generated in the rear of the model, especially from the sides of the trailer. Similar to the "Airbag", also this device may have shock absorbing features to be considered in the structural design phase. The "Boat-tail" devices (Figure 3-c) are designed to adjust the wake generated in the rear part of the trailer. They are currently installed on vehicles in the US, where larger maximum trailer sizes are allowed. The main issues with this device concern the stiffness of the boat-tail surfaces: in case of impact with a vehicle hitting another one with this device installed in the rear they may be dangerous as blades since they need to be realized using a resistant material. To ensure the access to the payload it is necessary that boat-tails are movable and to reduce the space requirements they may be foldable for parking purposes. The "Front trailer" device (Figure 3-d) is designed to move the separation on the front-side part of the trailer to a most suitable position located few centimeter above the surface of the trailer. It has a very small size and is positioned in a location that does not affects the access to payload and may have very low impact on the parking and storage of trailers.

When the boat tail or the fin or the airbag are installed on the trailer, the access to the payload is limited and some technical solution must be developed to have an access to the trailer inside without the need to uninstall the devices.

The increase of overall dimensions with respect to the standard truck-trailer configuration for each device is shown in Table 2. Especially the increase of the width and height are an issue for the safety of the roads (as an example the height is connected with tunnels and bridges height and the lane size are fixed).

Table 2. Percentage of increase of the overall vehicle's dimension.

\begin{tabular}{|l|c|c|c|}
\hline & \% lenght & \% width & \% height \\
\hline Airbag & 5.1 & 0.0 & 0.0 \\
\hline Fin & 4.8 & 0.0 & 0.0 \\
\hline Front trailer device & 0.0 & 1.6 & 0.8 \\
\hline Boat tail & 5.0 & 0.0 & 0.0 \\
\hline
\end{tabular}

In order to prevent injuries in case of crash with others vehicle no sharp angle devices are tested (except the boat-tail) and all of them are designed to be constructed using soft materials.

\section{NUMERICAL MODEL}

The computational meshes are generated using the meshing tool provided by open-source CFD framework Open-FOAM ${ }^{\circledR}$. The generated mesh is a fully Cartesian grid in the entire domain, with layers on the truck surface.

Different simulations are performed to optimize the computational domain dimension related to the physics of the phenomena especially in cross-wind. The computational domain (see Figure 4) is the optimum solution for both avoiding the blockage effect of the truck in the domain and decreasing the computational cost. 


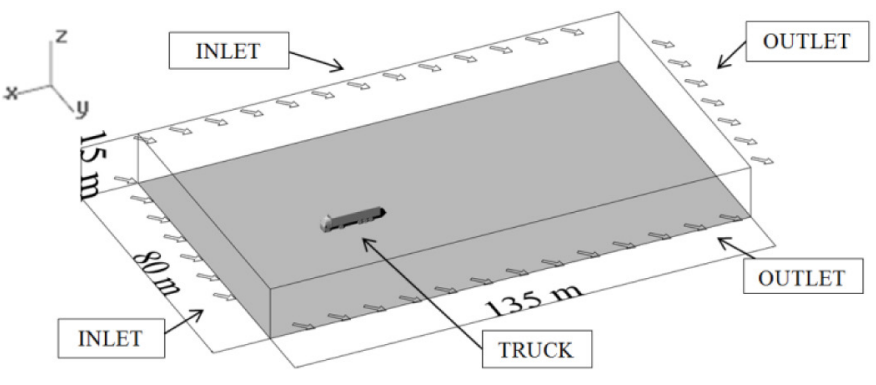

Figure 4. Computational domain and reference system.

The reference system is placed on the ground along the truck centerline. Positive $\mathrm{X}$ axis is directed according with the truck speed; positive $\mathrm{Z}$ axis is directed vertically and the $\mathrm{Y}$ axis has the truck transverse direction.

Two main rectangular volumetric controls, one inside the other are designed to refine the grid around the base geometry; other special refinement zones are defined to capture the wheel surface of the six tires. Several prismatic layers where added around the whole vehicle to correctly predict the flow in the boundary layer. Drag, side and lift force are computed as the force applied on the vehicle in $\mathrm{x}, \mathrm{y}$ and $\mathrm{z}$ direction according to the reference system in Figure 4. Truck trailer aerodynamic force coefficients are calculated according to the following:

$$
\begin{gathered}
C_{X}=-\frac{F_{X}}{0,5 * \rho * U_{\infty}^{2} * A_{\text {res }}} \quad C_{Y}=\frac{F_{Y}}{0,5 * \rho * U_{\infty}^{2} * A_{\text {res }}} \\
C_{Z}=\frac{F_{Z}}{0,5 * \rho * U_{\infty}^{2} * A_{\text {res }}}
\end{gathered}
$$

Where $\rho$ is the air density, $U_{\infty}$ is the speed of the air, $A_{R E S}$ is the frontal area of the vehicle for both front and crosswind.

Different grids from 10.8 million of elements to 48.5 million of elements are tested to check the grid independency on the target vehicle (see Table 3). The overall drag of the vehicle is used as parameter to monitor the grid independency in agreement with the SAE Standard J2966 [18]. The chosen grid, for the RANS simulations have around 10-11 million of elements (depending on the installed devices), the cells size around the truck is $0,015 \mathrm{~m}$ and the $\mathrm{y}+$ value is around 30 ; while the unsteady simulations are run with mesh of 40 million of elements. In Figure 5 some mesh details are shown, assessing the good mesh quality and the reproduction of the prismatic cell layers all over the truck and trailer surface.

In Figure 6 the mesh detail of the tested devices are reported for the mesh of 10 million of elements.

Table 3. Grid independency study.

\begin{tabular}{|c|c|c|}
\hline Million of elements & $\mathbf{C}_{\mathbf{X}}$ & $\mathbf{~}_{\mathbf{~}} \mathbf{C}_{\mathbf{X}}$ \\
\hline 10.8 & 0.586 & \\
\hline 39.7 & 0.581 & 0.86 \\
\hline 48.5 & 0.583 & 0.61 \\
\hline
\end{tabular}

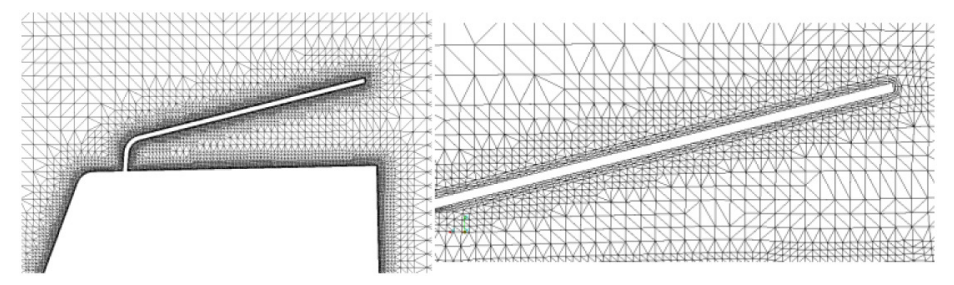

Figure 5. Mesh detail on the tractor top.


Figure 6. Mesh detail of the devices installed on the trailer: airbag geometry (a), fin geometry (b), boat tail (c), front trailer device (d)-

Time invariant incompressible RANS equations are solved using the simpleFoam [19] standard solver and the two equation k- $\omega$ SST turbulence model is used. Second-order upwind discretization scheme is used and SIMPLEC algorithm is adopted for coupling pressure and velocity [20].

The inlet velocity is set to $27 \mathrm{~m} / \mathrm{s}$; the ground has the same velocity of the inlet. This wind velocity generates a Reynolds Number higher than $7 \cdot 10^{6}$.

The truck is considered stationary while the flow and the ground have the relative velocity. The wheel of the truck and the trailer are put in rotation, in order to reproduce the operating condition of the truck. When analyzing only the devices performances, neglecting possible crosswind issues, the air speed (equal to the speed of the truck) is parallel to the $\mathrm{x}$ direction, zero pressure is set up at the outlet and slip wall is used for top and lateral boundaries. When cross wind effect are taken into account, the air velocity is setup in the $\mathrm{x}$ and $\mathrm{y}$ direction in both inlet and right side (where the $\mathrm{x}$-component is the truck speed and the y-component is the crosswind velocity), while zero pressure is set-up in outlet and left side. Slip wall are used for the top. Yaw angle from $0^{\circ}$ to $30^{\circ}$ with and angle step of $5^{\circ}$ are tested.

In order to speed up the simulation convergence and the computational time the steady state simulations are initialized with the potential solver potentialFoam [19]. RANS simulations are run until the convergence of the aerodynamic forces is reached. 
When time invariant simulations are performed, Detached eddy simulation (DES) with the one equation Spalart-Allmaras [20] near wall model is used, transient equations are solved using the standard pimpleFoam solver [19].

DES simulations are performed using a time step suitable for the mesh size around the vehicle, paying attention to keep the Courant number below 1 . The influence of different time steps were studied and the most suitable value for the time step is $10^{-4} \mathrm{~s}$. Unsteady calculations were performed until statistically stable aerodynamic forces were calculated on the truck and the trailer.

Yaw angles of $0^{\circ}, 5^{\circ}$ and $10^{\circ}$ are taken into account for the time variant simulations.

The set-up agrees with the SAE Standard J2966 [18].

\section{FRONT WIND ANALYSIS}

\section{Target Vehicle}

The Target vehicle has an overall drag of 0.586 ; the $14 \%$ of it is only generated from the wheels of the tractor and of the trailer.

From the normalized magnitude velocity contour plot in Figure 7, the region in which the main vortices are located is the rear of the trailer; the underbody part of it and the mirrors of the tractor. The vortices located in the rear of the trailer (see Figure 7 on the top) have different lengths: the top-rear vortex has a dimension almost double compared with the bottom-rear one, suggesting that it could be convenient to realize a device according with this non symmetric flow on the zero x-z plane. In Figure 7 on the bottom, the flow-field around the target vehicle is shown on the plane $x-y$ at $z=2.6 \mathrm{~m}$ where the mirror vortices and the side-rear ones are fully developed.

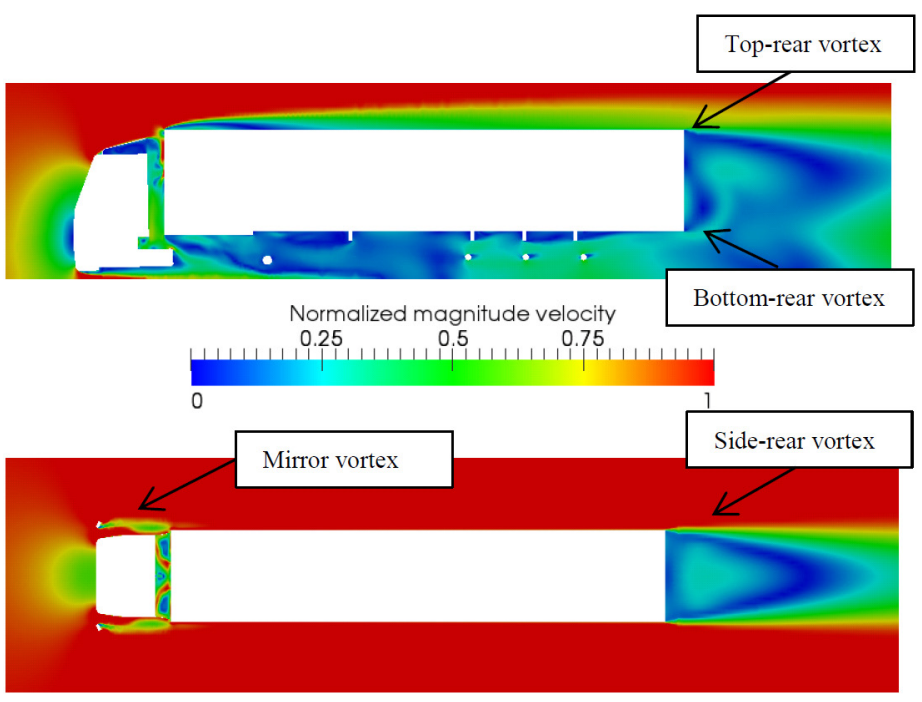

Figure 7. Normalized magnitude velocity contour plot. Top: vehicle centerline plane, bottom: horizontal plane at a height of $2.6 \mathrm{~m}$ from the ground.

\section{Airbag}

Airbags are designed to influence the rear of the truck vortices and are installed on the rear face of the truck; the airbag increases only the maximum length of the heavy truck ( $5 \%$ of the initial dimension). This rise in the overall length of the vehicle is not considered a big issue for the street safety. Especially if the device is made in soft material it will work also as an airbag in case of crash.

The main flow differences with respect to the target vehicle are shown in Figure 8: the bottom-rear vortex has a different shape compared to the one on the target vehicle, being largely reduced in length, leading to a drag reduction around 3.5\%.

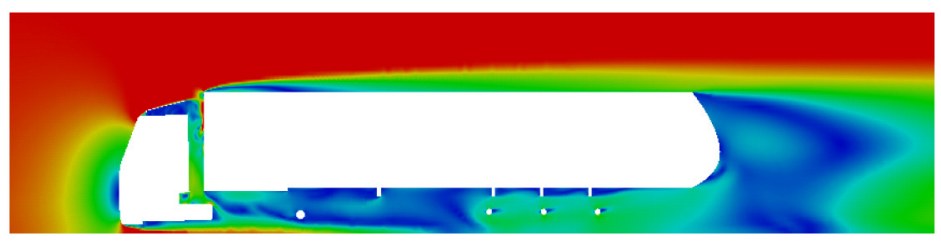

Figure 8. Normalized magnitude velocity contour plot at $\mathrm{y}=0 \mathrm{~m}$.

\section{Fin}

As mentioned before a suitable design of the bottom part of the fin is crucial to obtain a high drag reduction from the device. This device increases the length of the heavy truck of 5\% of the target vehicle length.

The fin shows high ability in reducing the overall heavy truck drag around the $8 \%$. In this case the drag reduction is more evident than for the airbags, indicating that using a device that influences the lateral vortex structures it is possible to enhance the drag reduction.

The main advantage of this kind of device compared to the airbag one is in the reduction of the overall size of the vortices in the rear of the truck. In Figure 9, it is possible to see that the wake behind the truck is reduced with respect to the target vehicle one and justifies the drag reduction that is obtained.

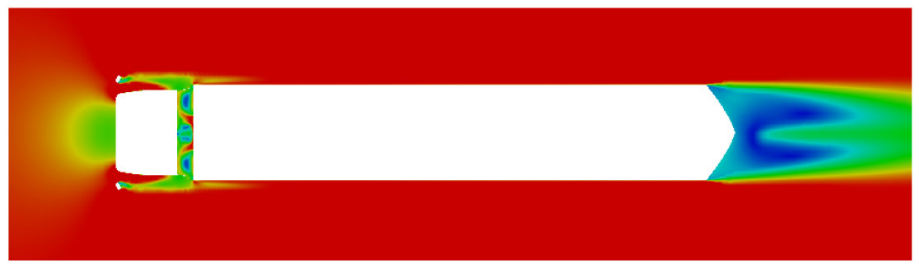

Figure 9. Normalized magnitude velocity contour plot at $\mathrm{z}=2.6 \mathrm{~m}$.

\section{Front Trailer Device}

This device is developed to minimize the impact of the device on the operation of the vehicle and to limit the costs of production compared to the previous devices and so promote their diffusion. It also guarantees an easy access to the payload, when the device is installed without the need to develop complex movement. Despite these benefits the front trailer device requires a minimum increase in the overall dimensions of the truck: about $1.6 \%$ increase of width and $0.8 \%$ increase in height, while no increase in the vehicle length is required. Both the raise in width and height may not be considered as an issue to the street safety. With a minimum increase of the overall dimension of 
the truck, it is possible to obtain a drag reduction of $3.55 \%$ due to the shift of the separation position in the top-front and sides-front parts of the trailer. The drag reduction is achieved despite of the increase of frontal area, nevertheless the frontal area increase is lower than $10 \%$. The comparison in terms of force coefficients is always performed using as reference area the frontal area of the target vehicle.

Due to the position of this device, it is possible to install it also in combination with one of the previous devices (airbag, fin or boat-tail) to have a larger decrease in the drag force on the heavy truck.

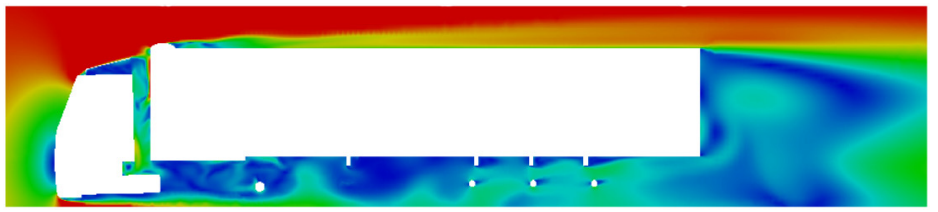

Figure 10. Normalized magnitude velocity contour plot at $\mathrm{y}=0 \mathrm{~m}$.

\section{Coupling Devices}

In the previous paragraphs different kinds of devices are tested, adding them, one after the other on the target vehicle.

As already said, the front trailer device can easily be added on the truck, when also other devices as the airbag or fin are mounted on the rear of the trailer. For this reason different mix of devices are tested: the front trailer device with the airbag and the front trailer device with the fin.

The results of these simulations are reported in Table 4.

Table 4. $\mathrm{C}_{\mathrm{X}}$ coefficient reduction.

\begin{tabular}{|c|c|c|}
\hline & $\mathbf{C}_{\mathbf{X}}$ & $\mathbf{\%}_{\mathbf{~}} \mathbf{C}_{\mathbf{X}}$ reduction \\
\hline Target vehicle + airbag & 0.560 & 3.52 \\
\hline Target vehicle + fin & 0.540 & 7.87 \\
\hline $\begin{array}{c}\text { Target vehicle + front trailer device }+ \\
\text { airbag }\end{array}$ & 0.519 & 11.46 \\
\hline Target vehicle + front trailer device + fin & 0.558 & 4.82 \\
\hline
\end{tabular}

Coupling the front trailer device with the airbag the drag force strongly decreases: more than $11 \%$ reduction is obtained with a reasonable raise in the overall truck dimension. On the other hand, when the front trailer device is installed together with the fin, a negative effect on the overall vehicle drag occurs, suggesting that the fin require more free-stream flow-field from the side part of the trailer.

\section{DEVICES DIMENSION OPTIMIZATION}

After this preliminary study, an extended optimization of the shape of the previous devices is performed in order to maximize the drag reduction. This procedure first requires a parametrization of the shape of the device respect at a suitable parameter, chosen as the length of the device, due to its influence on the overall dimension of the vehicle. The parameter is changed in a suitable range to make a family of devices with the same characteristics. The optimization is performed with the set-up previously presented. The definition of only one parameter for the optimization of the shape of the device is considered enough to find a rough optimum while a more detailed analysis should be done especially if one solution is going to be studied with more detail.

As previously mentioned this optimization is performed only for the airbag and for the front trailer device due to the poor performance of the fin when is coupled with the front trailer device.

The results of this optimization are compared with the results obtained by the boat tail length optimization.
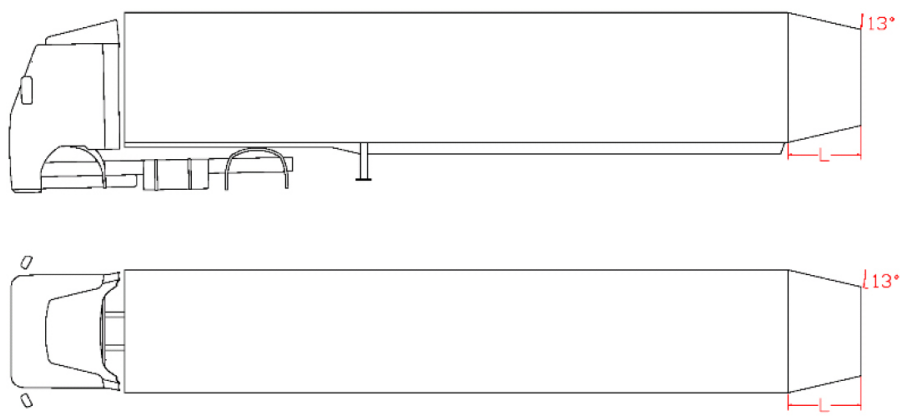

Figure 11. Boat tail parameters used for the optimization: side view (top), top view (bottom).

The optimization of the boat tail and of the airbag are performed using the length "L" of the device as the "leading parameter" (see Figures 11, 12). In literature there are no certain agreements concerning the optimum length of this device. Cooper [10] observed an optimum length of the device around $0.6 \mathrm{~m}$, Croll et al. [21] showed a benefit in increasing the length of the device up to $2.4 \mathrm{~m}$, while, in Salari [22] measurements, no reasonable improve of drag reduction are observed when the length of the device is increased from $0.8 \mathrm{~m}$ to $1.2 \mathrm{~m}$.

Table 5. $\mathrm{C}_{\mathrm{X}}$ coefficient reduction as a function of length $\mathrm{L}$ of the boat-tail.

\begin{tabular}{|c|c|c|}
\hline $\mathbf{L}[\mathbf{m}]$ & $\mathbf{C}_{\mathbf{X}}$ & $\boldsymbol{\%}_{\mathbf{X}} \mathbf{C}_{\mathbf{X}}$ reduction \\
\hline 0.25 & 0.535 & 8.620 \\
\hline 0.5 & 0.540 & 7.896 \\
\hline 0.75 & 0.542 & 7.522 \\
\hline 1 & 0.546 & 6.837 \\
\hline 1.25 & 0.542 & 7.443 \\
\hline 1.5 & 0.536 & 8.473 \\
\hline 1.75 & 0.537 & 8.431 \\
\hline 2 & 0.533 & 9.068 \\
\hline
\end{tabular}

The angle between the boat tail and the side and the top of the device is fixed at $13^{\circ}$ following Browand's recommendations (Browand [23]). All boat-tail lengths show their ability in reducing the drag of the overall vehicle (see Figure 12 and Table 5), nevertheless the most suitable one is the smallest: it guarantees a good drag reduction compared to the other lengths and it will be the easier one to design and to manufacture on the trailer. The red dashed line in Figure 12 , $\underline{13}$, and 14 represents the $\mathrm{C}_{\mathrm{X}}$ of the target vehicle. The results agree in 
general with previous works: in this case the trend is not monotone and the minimum in drag reduction is around the length of the device of $1 \mathrm{~m}$ and for larger lengths the drag decreases again.

\section{$\mathrm{Cx}$}

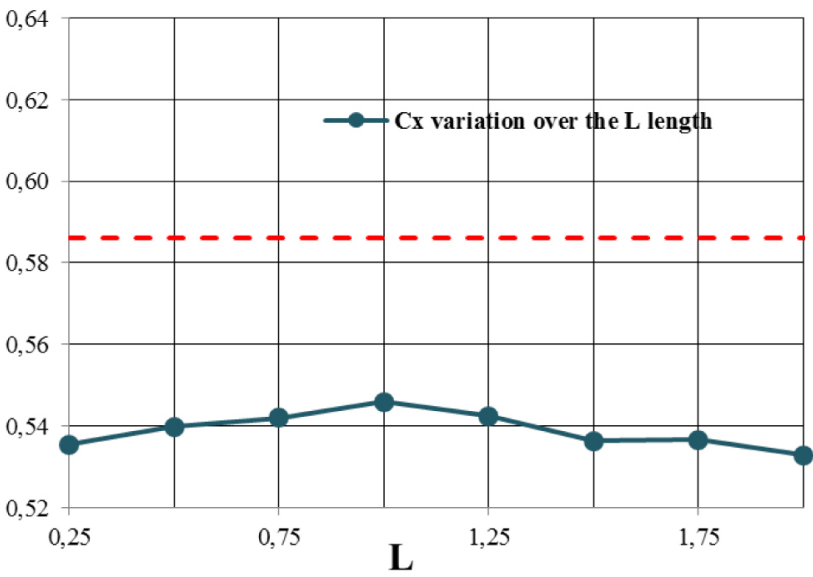

Figure 12. Boat tail $C_{X}$ coefficient reduction as a function of length $L$ of the boat-tail. The dashed line represents the drag of the target vehicle.

For the airbag (see Figure 13) it is chosen the total length of the airbag as optimization parameter. It is possible to find a relative maximum of drag reduction at the length $\mathrm{L}=0.75 \mathrm{~m}$ (see Figure 14).

This is considered the optimum length for this device considering also is dual function: it may work as an airbag in case of crash. Length larger than $0.75 \mathrm{~m}$ are not considered convenient for drag reduction purposes.
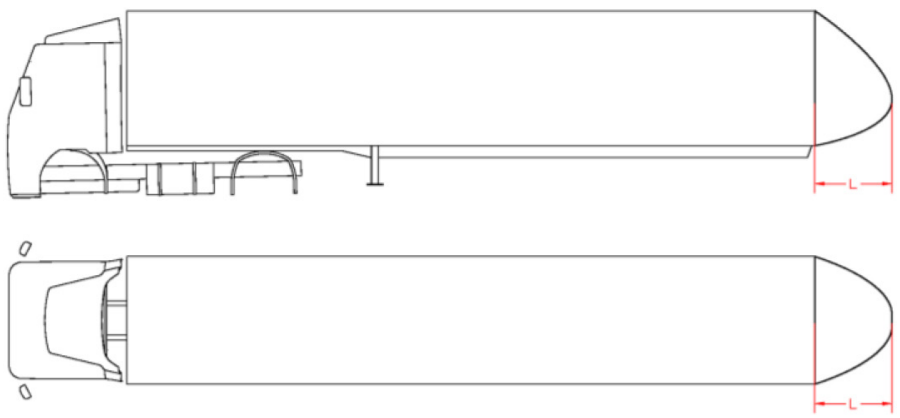

Figure 13. Airbag parameters used for the optimization: side view (top), top view (bottom).

Table $6 . \mathrm{C}_{\mathrm{X}}$ coefficient reduction as a function of length $\mathrm{L}$ of the airbag.

\begin{tabular}{|c|c|c|}
\hline $\mathbf{L}[\mathbf{m}]$ & $\mathbf{C}_{\mathbf{X}}$ & $\% \mathbf{C}_{\mathbf{X}}$ reduction \\
\hline 0.25 & 0.590 & -0.637 \\
\hline 0.5 & 0.575 & 1.884 \\
\hline 0.75 & 0.568 & 3.040 \\
\hline 1 & 0.569 & 2.816 \\
\hline 1.25 & 0.576 & 1.683 \\
\hline 1.5 & 0.577 & 1.501 \\
\hline 1.75 & 0.567 & 3.303 \\
\hline 2 & 0.545 & 6.988 \\
\hline
\end{tabular}

$\mathbf{C x}$

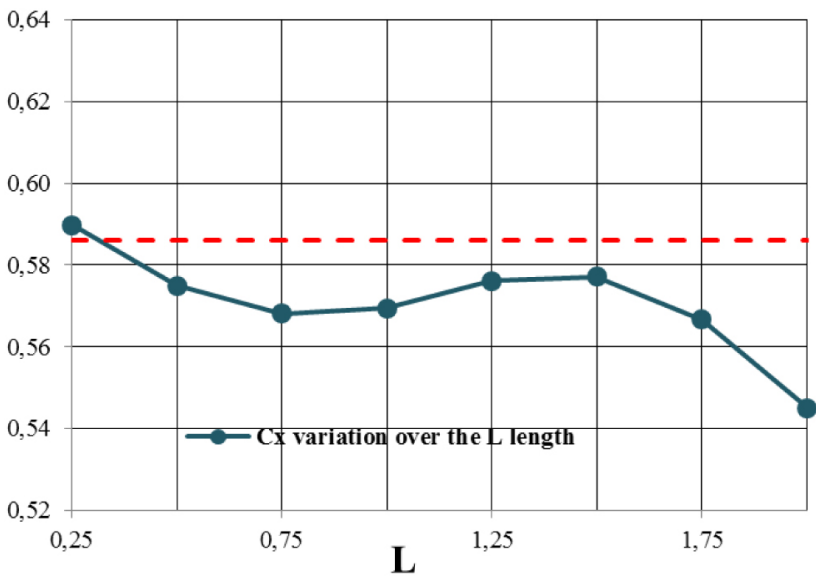

Figure 14. Airbag $C_{X}$ coefficient reduction as a function of length $L$ of the airbag. The dashed line represents the drag of the target vehicle.

The front trailer optimization is performed considering a small device due to safety issue and to match the current regulations (Directive 96/53/EC [3] ) concerning the maximum size allowed for a heavy truck (see Figure 15). The height of the device is fixed at $b=5 \mathrm{~cm}$ while the length $a$ is the "leading" parameter for the optimization (device shape patent of Politecnico di Milano 2014 [24] ).

In this way the percentage increase in height is about $0.3 \%$ and in width around $0.6 \%$, corresponding to an increase of the frontal area of $5 \%$. When the length " $a$ " (see Figure 15) of this device is lower than $0.1 \mathrm{~m}$ the drag increases while with larger lengths the drag strongly decreases achieving a convergence behavior and a maximum in drag reduction around 6\% (see Figure 16). This device, compared to the other ones, can be installed on any truck (previous, next, current generation) without any impact on the functionality of the vehicle and without any safety issue.
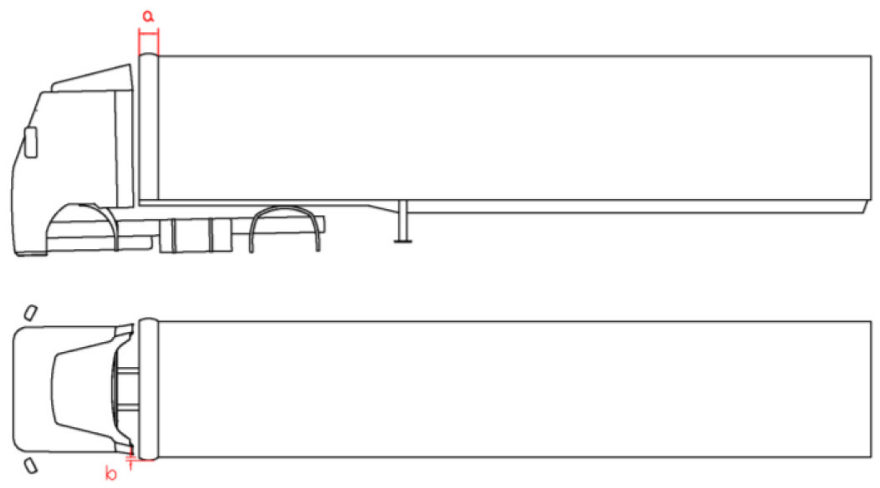

Figure 15. Front trailer device optimization's parameter: side view (top), top view (bottom).

Another crucial aspect is the possibility to install more than one front trailer device along the trailer length to exploit the possibility to achieve a higher impact on the vehicle drag. A number of devices between 1 and 5, with "a" dimension set to $0.35 \mathrm{~m}$, are located at equal distance from each other starting from two devices: one on the front part of the trailer and one in the rear part of it (see Figure 17). The results in Table 5 show a maximum drag reduction around $11.93 \%$ that is more the double compared to the single device case, 
and it is reached when two devices are installed on the vehicle: one in the front part and one in the rear part. When two devices of length $\mathrm{a}=0.45 \mathrm{~m}$ are installed on the trailer it is achieved a minimum in drag coefficient of 0.511 corresponding to a drag reduction of $12.85 \%$. This is achieved because the operation of the devices is double: one is the separation of the flow at the front of the trailer and the second result is a reduction of the trailer wake in the back.

Table 7. $\mathrm{C}_{\mathrm{X}}$ coefficient reduction as a function of length a of the front trailer device.

\begin{tabular}{|c|c|c|}
\hline $\mathbf{a}[\mathbf{m}]$ & $\mathbf{C}_{\mathbf{X}}$ & \% $\mathbf{C}_{\mathbf{X}}$ reduction \\
\hline 0.05 & 0.638 & -8.952 \\
\hline 0.1 & 0.604 & -3.150 \\
\hline 0.15 & 0.576 & 1.632 \\
\hline 0.2 & 0.565 & 3.515 \\
\hline 0.25 & 0.558 & 4.719 \\
\hline 0.3 & 0.556 & 5.183 \\
\hline 0.35 & 0.554 & 5.440 \\
\hline 0.4 & 0.556 & 5.099 \\
\hline 0.45 & 0.550 & 6.131 \\
\hline
\end{tabular}

\section{$\mathbf{C x}$}

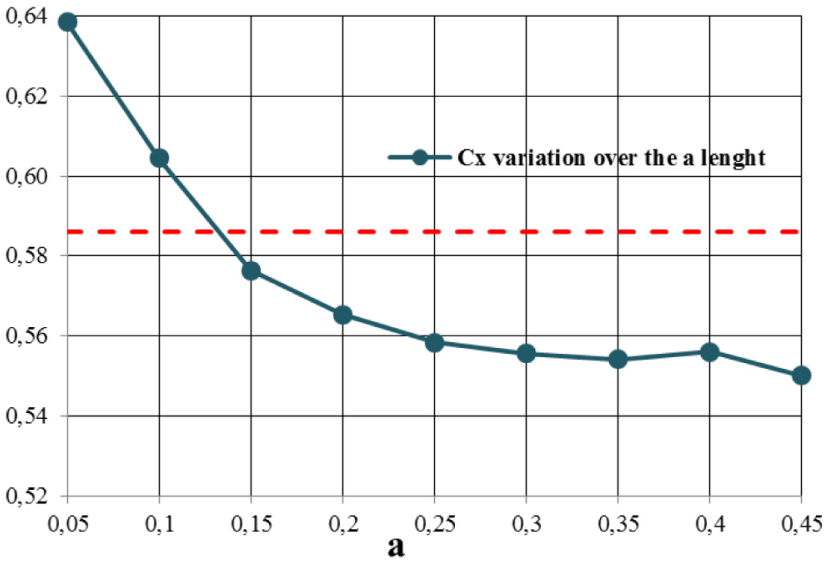

Figure 16. Front trailer $\mathrm{C}_{\mathrm{X}}$ coefficient reduction as a function of length a of the front trailer device. The dashed line represents the drag of the target vehicle.
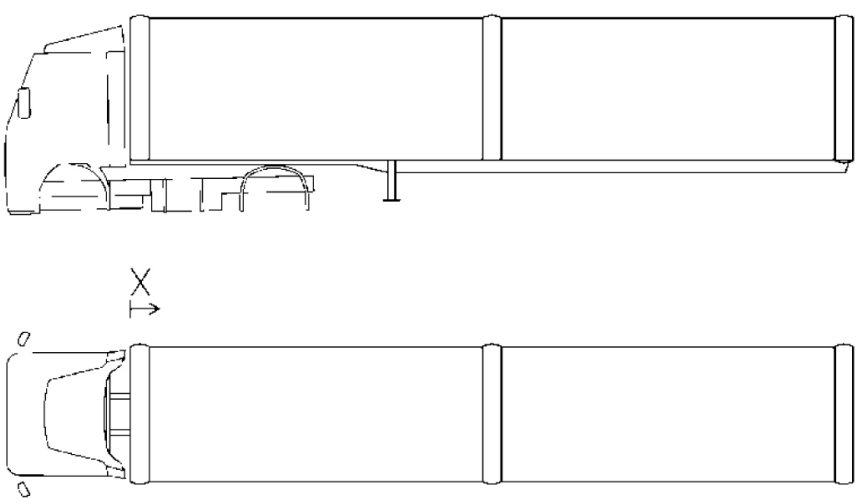

Figure 17. Three front trailer device installed on the trailer.
Table 8. Number of front trailer device installed on the trailer drag coefficient reduction.

\begin{tabular}{|c|c|c|}
\hline Number of front trailer device installed & $\mathbf{C}_{\mathbf{X}}$ & $\mathbf{\%} \mathbf{C}_{\mathbf{X}}$ reduction \\
\hline 1 & 0.554 & \\
\hline 2 & 0.516 & 11.927 \\
\hline 3 & 0.520 & 11.233 \\
\hline 4 & 0.522 & 10.985 \\
\hline 5 & 0.525 & 10.452 \\
\hline
\end{tabular}

\section{CROSS-WIND}

In order to test the performance of the designed devices also in cross-wind conditions some additional calculations are performed investigating the yaw angle range from $0^{\circ}$ to $30^{\circ}$ with steps of 5 degrees.

The cross-wind study are performed with the following devices:

- $\quad$ Airbag $(\mathrm{L}=0,75 \mathrm{~m})$, see Figure 13 ;

- $\quad$ Front trailer device $(\mathrm{a}=0,45 \mathrm{~m})$, see Figure 15 ;

- Two Front trailer device located one in the front of the trailer and one in rear of it $(\mathrm{a}=0.45 \mathrm{~m})$, called "front-rear trailer device".

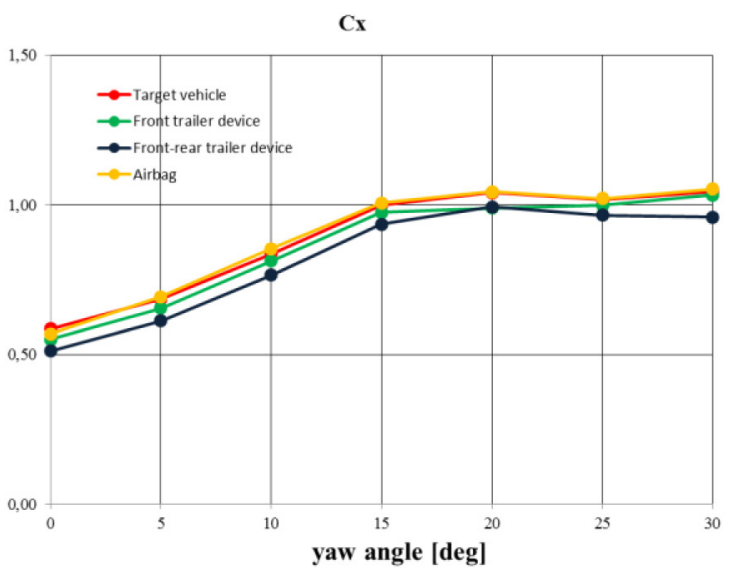

Figure $18 . \mathrm{C}_{\mathrm{X}}$ variation over the yaw angle for the truck, without any device, with the front trailer device, with the front-rear trailer device and with the airbag.

Table 9. $\mathrm{C}_{\mathrm{X}}$ variation over the yaw angle for the truck, without any device, with the front trailer device, with the front-rear trailer device and with the airbag.

\begin{tabular}{|c|c|c|c|}
\cline { 2 - 4 } \multicolumn{1}{c|}{} & Target vehicle & \multicolumn{2}{c|}{ Front trailer device } \\
\hline yaw angle & $\mathbf{C}_{\mathbf{X}}$ & $\mathbf{C}_{\mathbf{X}}$ & $\mathbf{C}_{\mathbf{X}} \%$ reduction \\
\hline 0 & 0.586 & 0.550 & $6.13 \%$ \\
\hline 5 & 0.684 & 0.653 & $4.47 \%$ \\
\hline 10 & 0.836 & 0.812 & $2.80 \%$ \\
\hline 15 & 1.000 & 0.975 & $2.51 \%$ \\
\hline 20 & 1.040 & 0.990 & $4.86 \%$ \\
\hline 25 & 1.016 & 0.998 & $1.82 \%$ \\
\hline 30 & 1.044 & 1.033 & $1.09 \%$ \\
\hline
\end{tabular}


Table 9. (cont.) $\mathrm{C}_{\mathrm{X}}$ variation over the yaw angle for the truck, without any device, with the front trailer device, with the front-rear trailer device and with the airbag.

\begin{tabular}{|c|c|c|c|c|}
\cline { 2 - 5 } \multicolumn{1}{c|}{} & \multicolumn{2}{c|}{ Front-rear trailer device } & \multicolumn{2}{c|}{ airbag } \\
\hline yaw angle & $\mathbf{C}_{\mathbf{X}}$ & $\mathbf{C}_{\mathbf{X}} \%$ reduction & $\mathbf{C}_{\mathbf{X}}$ & $\mathbf{C}_{\mathbf{X}} \%$ reduction \\
\hline 0 & 0.511 & $12.79 \%$ & 0.568 & $3.04 \%$ \\
\hline 5 & 0.612 & $10.52 \%$ & 0.693 & $-1.26 \%$ \\
\hline 10 & 0.765 & $8.45 \%$ & 0.853 & $-2.04 \%$ \\
\hline 15 & 0.935 & $6.51 \%$ & 1.007 & $-0.67 \%$ \\
\hline 20 & 0.993 & $4.52 \%$ & 1.043 & $-0.27 \%$ \\
\hline 25 & 0.965 & $5.05 \%$ & 1.021 & $-0.47 \%$ \\
\hline 30 & 0.959 & $8.12 \%$ & 1.052 & $-0.80 \%$ \\
\hline
\end{tabular}

The $\mathrm{C}_{\mathrm{X}}$ variation over the yaw angle is shown in Figure 18. In general the $C_{X}$ raises with the yaw angle until a value around 15 degrees after which it stays almost constant as in the work of Das et al. [25] Especially the front-rear trailer device shows a general good behavior at any of the tested yaw angle suggesting its suitability for both front and crosswind.

The simulation performed on the airbag shows that this solution does not guarantee the $\mathrm{C}_{\mathrm{X}}$ reduction also in crosswind conditions.

Nevertheless this shape is generated from a one parameter optimization based on the length of the device. The authors believe that with a different choice of parameter for the optimization, or considering more than one parameter, the results of the airbag device in crosswind can be positive as in front wind.

The variation of the $\mathrm{C}_{\mathrm{Y}}$ coefficient and $\mathrm{C}_{\mathrm{Z}}$ are shown for the target vehicle and for the previous tested devices in Figures 19 and $\underline{20}$. The more the yaw angle increases more the $\mathrm{C}_{\mathrm{Z}}$ rises; at the yaw angle between 5 and 10 degrees the $\mathrm{C}_{Z}$ turns from a downforce a up force as observed also by Das et al. [25]. The side force coefficient linearly increases from almost a value similar to zero in front-wind as in the work presented by Das et al. [25] .

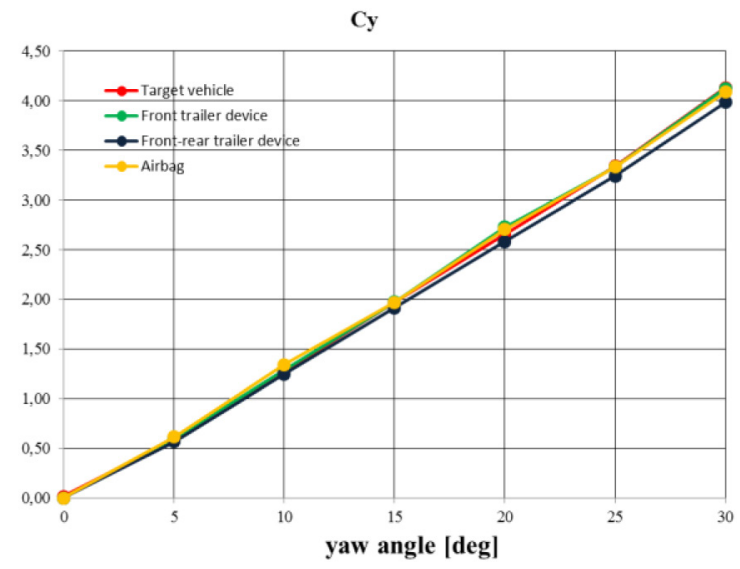

Figure 19. $C_{\mathrm{Y}}$ variation over the yaw angle for the truck, without any device, with the front trailer device, with the front-rear trailer device and with the airbag.

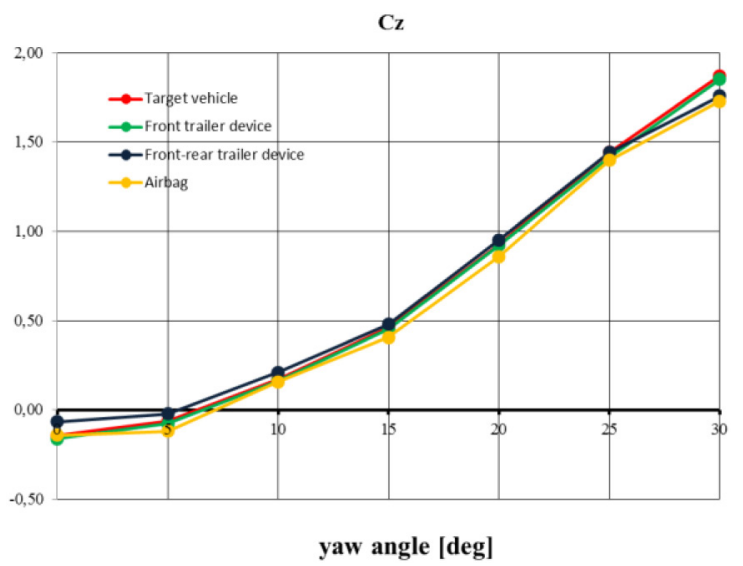

Figure $20 . C_{Z}$ variation over the yaw angle for the truck, without any device, with the front trailer device, with the front-rear trailer device and with the airbag.

\section{DES SIMULATIONS - ZERO YAW ANGLE}

For the unsteady simulation a mesh of about 40 millions of elements is used to achieve a satisfying flow detail. Starting from the solution of the flow in the steady-state simulation it is possible to obtain a statistically stable solution in 5000 time steps. Lift, drag, side force fluctuate no more than \pm 5 percent of the average value. The time average is performed analyzing 25000 time steps after reaching the convergence in the shedding vortices.

The main sources of drag for the target truck are indicated in the contour plots of velocity (Figure 21-a, 22-a, 23-a.

The main separation region occurs in top-front of the trailer (indicated in Figure 21-a as "top-front vortex": this generates vortices that move along the top of the trailer with a consistent generation of drag.

Another important area, for the vortices propagation, is the rear of the trailer: two main vortices are generated in this area, one from the top-rear of the trailer and another one from the bottom-rear part of it (see Figure 21-a. The velocity of the flow, coming from the bottom-rear corner of the trailer, is almost the double of the one coming from the top rear one (the top flow is almost in free-stream while the bottom one comes from the very turbulent region in the underbody of the vehicle; for this reason the top-rear vortex has a dimension almost double compare to the bottom one.

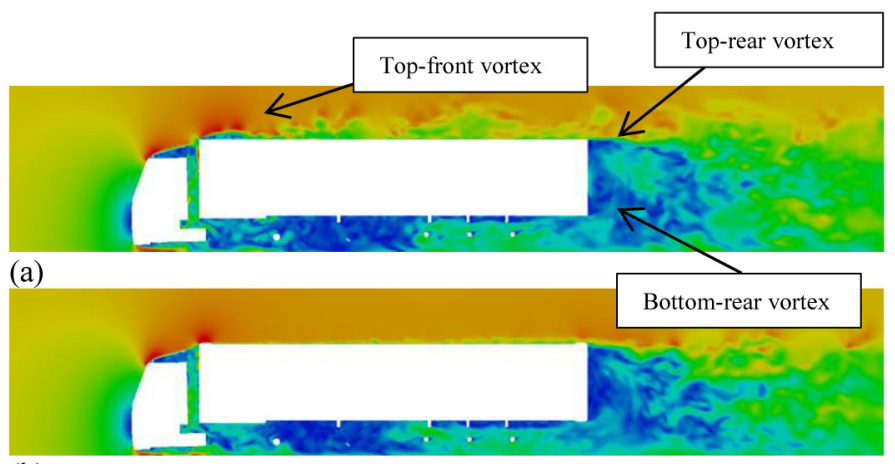

(b)

Figure 21. Instantaneous velocity contour plot at $\mathrm{y}=0 \mathrm{~m}$ : (a) target vehicle, (b) front-rear trailer device. 
Analyzing the top view of the vehicle (Figure 22-a) other important separation regions can be visualized: the first separation occurs on the side mirrors generating high frequency vortex shedding; a second separation is generated from the side of the truck: thanks to the side fairings installed on the truck, these two vortices move along the side surface of the trailer with a consistent generation of drag. Due to the box shape of the trailer, at the end of it, two main side-rear vortices are generated with almost the same size and frequency but with opposite phases.

All the results agree with the ones previous showed for the mesh around 10 million of elements (see Figure 7).

When the target vehicle is equipped with the front-rear trailer device a consistent reduction of drag around $12.5 \%$ is achieved in both RANS and DES simulation on the mesh of 40 million of elements (see Table 10).

The main differences in the flow-field between the target vehicle and the truck with the front-rear trailer device installed can be especially seen in the contour plot in Figure 21. When the devices are installed, on the top-front of the trailer, the main separation region in this area does not occur: the presence of the devices organize the flow that does not develop large vortex structures, but tends to be more organized. The smaller turbulent content moving along the top of the trailer decreases the overall drag of the vehicle.

The more organized flow that arrives on the device located in the rear part of the trailer; the wake of the trailer is smaller than the one of the target vehicle (bottom, rear, side vortices are smaller), decreasing the overall vehicle drag.

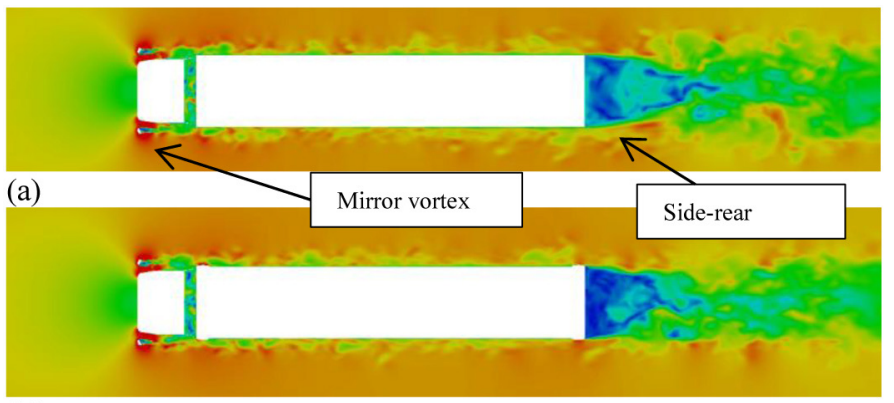

(b)

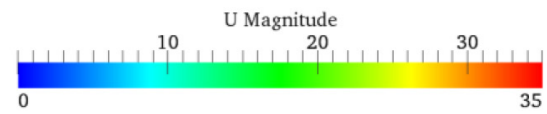

Figure 22. Instantaneous velocity contour plot at $\mathrm{z}=2.6 \mathrm{~m}$ : (a) target vehicle, (b) front-rear trailer device.

From the top view of the vehicle (see Figure 22), the vortices generated from the side mirrors mix with the vortices generated from the sides of the truck, as in the target vehicle, and then impact on the front-trailer device. This interaction produce smaller vortices moving along the sides of the trailer; in other x-y planes without the side mirrors this effect is better visible as in the Figure 21. The previous consideration is also demonstrated from the frequency analysis of the signals of drag, lift and side force. When the devices are installed on the vehicle the energy contribute of the signals is more distributed along a larger range of frequency compare to the target vehicle.

(a)

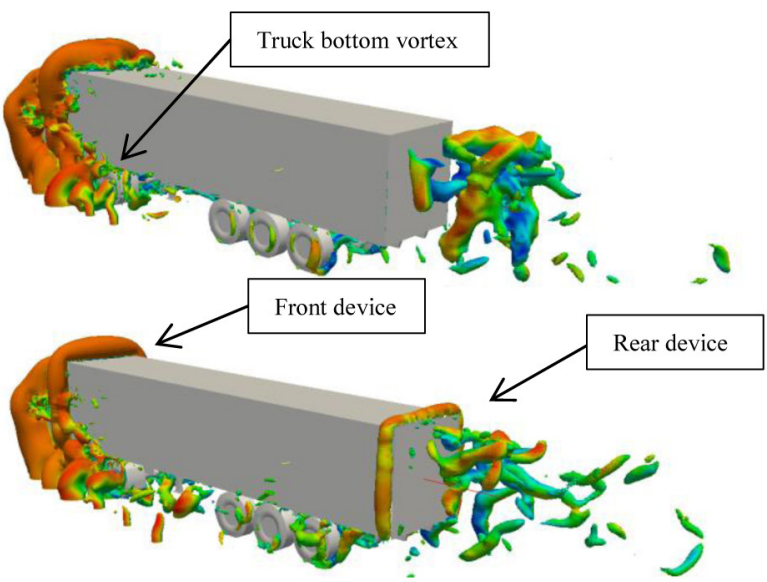

Figure 23. Instantaneous vorticity: (a) target vehicle, (b) front-rear trailer device.

All the previous considerations concerning the difference between the target vehicle and the one with the devices mounted on it are shown in the Figure 23, where the instantaneous vorticity surfaces with a constant value of 100 are compared and colored according to the local air speed magnitude.

The main differences between the simulations are in the regions where the devices are installed. In the target vehicle, the top-front part of the trailer as a higher vorticity compared to the same region when the front device is mounted. In the top-rear part of the trailer in the target vehicle the flow-field is almost in free stream, while the vorticity rises when the rear device is installed. Another crucial area for the drag reduction is the bottom part of the truck but the front-rear trailer device do not influence it. In general when the devices are installed the vortex structures appear to be more organized than for the target vehicle.

Table 10. $C_{X}, C_{Y}, C_{Z}$ coefficient of the target vehicle as a function of the yaw angle.

Target vehicle
\begin{tabular}{|c|c|c|}
\hline yaw angle & $\mathbf{C}_{\mathbf{X}}$ RANS & $\mathbf{C}_{\mathbf{X}}$ DES \\
\hline 0 & 0,581 & 0,623 \\
\hline 5 & 0,661 & 0,679 \\
\hline 10 & 0,824 & 0,813 \\
\hline
\end{tabular}

\begin{tabular}{|c|c|c|}
\hline yaw angle & $\mathbf{C}_{\boldsymbol{Z}}$ RANS & $\mathbf{C}_{\boldsymbol{Z}}$ DES \\
\hline 0 & $-0,155$ & $-0,105$ \\
\hline 5 & $-0,058$ & $-0,048$ \\
\hline 10 & 0,188 & 0,183 \\
\hline
\end{tabular}

\begin{tabular}{|c|c|c|}
\hline yaw angle & $\mathbf{C}_{\mathbf{Y}}$ RANS & $\mathbf{C}_{\mathbf{Y}}$ DES \\
\hline $\mathbf{5}$ & 0,594 & 0,616 \\
\hline 10 & 1,307 & 1,379 \\
\hline
\end{tabular}


Table 11. $\mathrm{C}_{\mathrm{X}}, \mathrm{C}_{\mathrm{Y}}, \mathrm{C}_{\mathrm{Z}}$ coefficient over the yaw angle of the vehicle with the front-rear trailer installed.

Front-rear trailer device
\begin{tabular}{|c|c|c|c|c|}
\hline $\begin{array}{c}\text { yaw } \\
\text { angle }\end{array}$ & $\begin{array}{c}\mathbf{C}_{\mathbf{X}} \\
\text { RANS }\end{array}$ & $\begin{array}{c}\%_{\mathbf{X}} \mathbf{C}_{\mathbf{X}} \text { reduction } \\
\text { RANS }\end{array}$ & $\begin{array}{c}\mathbf{C}_{\mathbf{X}} \\
\text { DES }\end{array}$ & $\begin{array}{c}\text { \% }_{\mathbf{X}} \text { reduction } \\
\text { DES }\end{array}$ \\
\hline 0 & 0,508 & $12,506 \%$ & 0,544 & $12,702 \%$ \\
\hline 5 & 0,585 & $11,504 \%$ & 0,600 & $11,610 \%$ \\
\hline 10 & 0,753 & $8,606 \%$ & 0,753 & $7,454 \%$ \\
\hline
\end{tabular}

\begin{tabular}{|c|c|c|c|c|}
\hline $\begin{array}{c}\text { yaw } \\
\text { angle }\end{array}$ & $\begin{array}{c}\mathbf{C}_{\mathbf{Y}} \\
\text { RANS }\end{array}$ & $\begin{array}{c}\text { \% } \mathbf{C}_{\mathbf{Y}} \text { reduction } \\
\text { RANS }\end{array}$ & $\begin{array}{c}\mathbf{C}_{\mathbf{Y}} \\
\text { DES }\end{array}$ & $\begin{array}{c}\text { \% } \mathbf{C}_{\mathbf{Y}} \text { reduction } \\
\text { DES }\end{array}$ \\
\hline 5 & 0,572 & $3,716 \%$ & 0,606 & $1,603 \%$ \\
\hline 10 & 1,265 & $3,202 \%$ & 1,364 & $1,066 \%$ \\
\hline
\end{tabular}

\begin{tabular}{|c|c|c|c|c|}
\hline $\begin{array}{c}\text { yaw } \\
\text { angle }\end{array}$ & $\begin{array}{c}\mathbf{C}_{\mathbf{Z}} \\
\text { RANS }\end{array}$ & $\begin{array}{c}\text { \% }_{\mathbf{Z}} \text { reduction } \\
\text { RANS }\end{array}$ & $\begin{array}{c}\mathbf{C}_{\mathbf{Z}} \\
\text { DES }\end{array}$ & $\begin{array}{c}\text { \% }_{\mathbf{Z}} \text { reduction } \\
\text { DES }\end{array}$ \\
\hline 0 & $-0,116$ & $-8,473 \%$ & $-0,085$ & $19,628 \%$ \\
\hline 5 & $-0,017$ & $70,650 \%$ & $-0,023$ & $51,738 \%$ \\
\hline 10 & 0,196 & $-3,777 \%$ & 0,187 & $-2,118 \%$ \\
\hline
\end{tabular}

\section{DES SIMULATIONS - CROSS WIND}

Time average drag, side and lift coefficient are reported in table 10 and 11 for the target vehicle and the one with the front-rear trailer device installed on it, at the tested yaw angle, for the mesh on the order of 40 million of elements.

$\mathrm{C}_{\mathrm{X}}, \mathrm{C}_{\mathrm{Y}}, \mathrm{C}_{\mathrm{Z}}$ coefficients in cross-wind, as predicted from previous work, as the one of Das et al. [르] , increase when the yaw angle rise. Especially the lift change from a negative values, while the yaw angle is set to zero, to a positive one, while the yaw angle rise up to 7-8 degrees (see Table 10, 11 and Figure 24).

The time averaged coefficients of the DES simulation are consistent with the results of the RANS simulation and generally are higher than the corresponding RANS simulations. In general all results obtained with the mesh of around 40 million of elements both in RANS and DES simulations agree with the results of the coarse mesh previous showed (see Figure 18, 19, 20).

When the front rear-trailer devices are installed on the truck; at every tested yaw angle $C_{X}$ is consistently observed to be reduced between 8 and 12 percent. The main differences between the simulations are showed in the prediction of the $\mathrm{C}_{\mathrm{Z}}$ (see Table 11). Due to the small values of the magnitude of the lift forces $[\mathrm{N}]$ when the yaw angle is between 0 and 5 degrees: a small perturbation of the lift force around $100 \mathrm{~N}$ (around $10 \mathrm{~kg}$ ) produces consistent changes in the lift even if, for this kind of problems (aerodynamics of heavy truck) this quantity is negligible.

The $\mathrm{C}_{\mathrm{Y}}$ force strongly increases when the yaw angle rises with no main difference between the simulations (see Figure 25).

In the next figure the contour plot of absolute velocity are shown. In the simulation the wind is coming from the front and left side of the truck (from the left and bottom part of each contour plot in the Figure 27). Increasing the yaw angle, the turbulence in the right-side part of the truck raises (top part of each contour plot in Figure 27) consistently increase the $\mathrm{C}_{\mathrm{X}}$. When the front-rear trailer device is installed on the truck, the vortices in the right-side of the truck decrease their dimension, decreasing the overall drag of the vehicle compared to the target vehicle. As previously mentioned, this positive effect of the device is also showed in the frequency analysis of the signals of drag, lift and side force. When the devices are installed on the vehicle the energy contribution of the signals is more distributed along a larger range of frequency also in cross-wind.

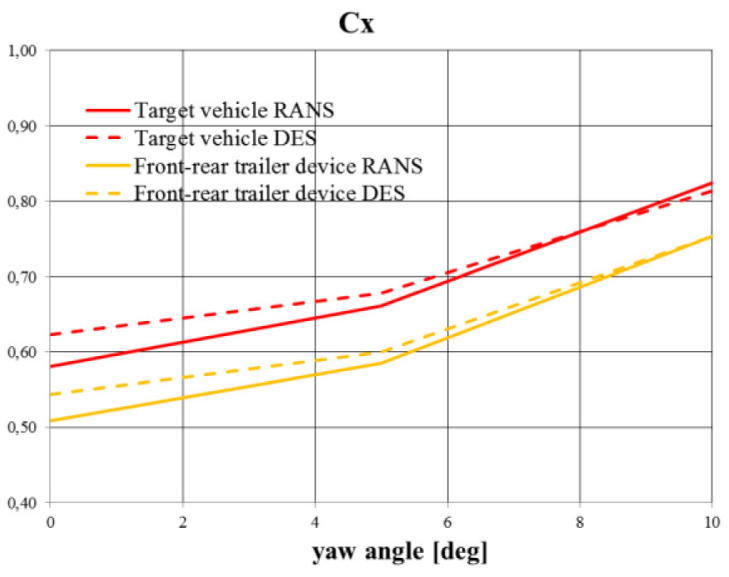

Figure 24. $C_{X}$ variation over the yaw angle for the truck, without any device, and with the front-rear trailer device installed.

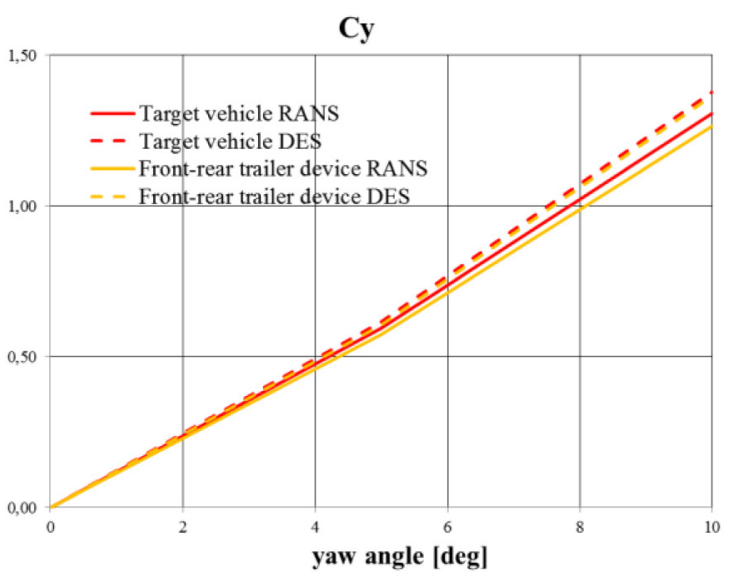

Figure 25. $\mathrm{C}_{\mathrm{Y}}$ variation for the target vehicle as a function of the yaw angle without any device and with the front-rear trailer device installed.

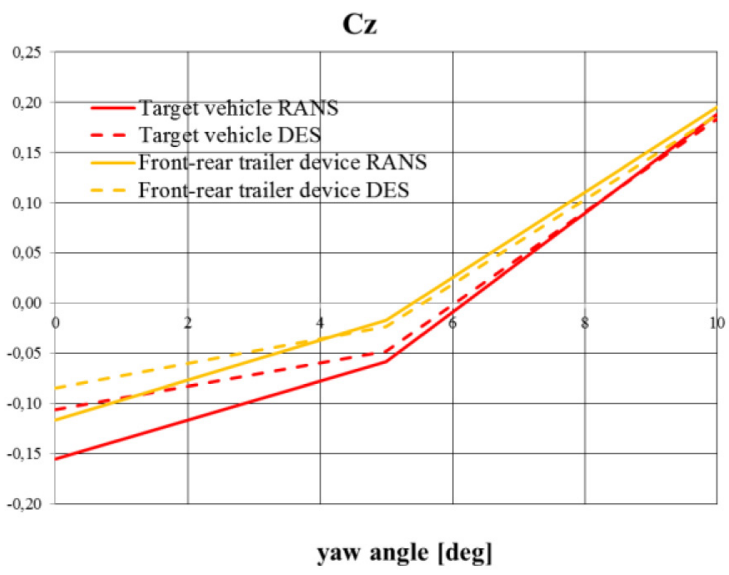

Figure 26. $C_{\mathrm{Z}}$ variation for the target vehicle as a function of the yaw angle without any device and with the front-rear trailer device installed. 


\section{Target vehicle}
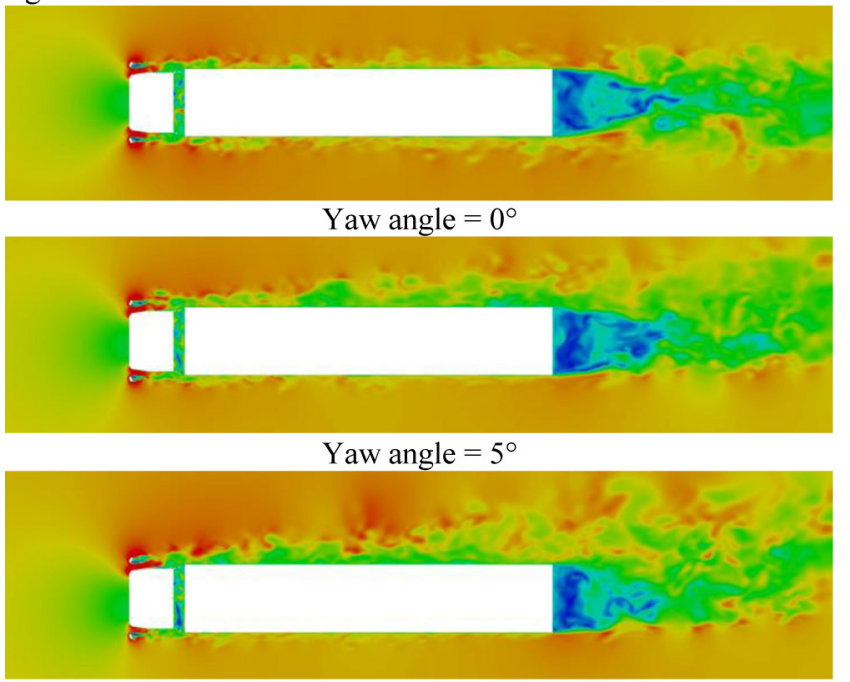

Yaw angle $=10^{\circ}$

Front-rear trailer device
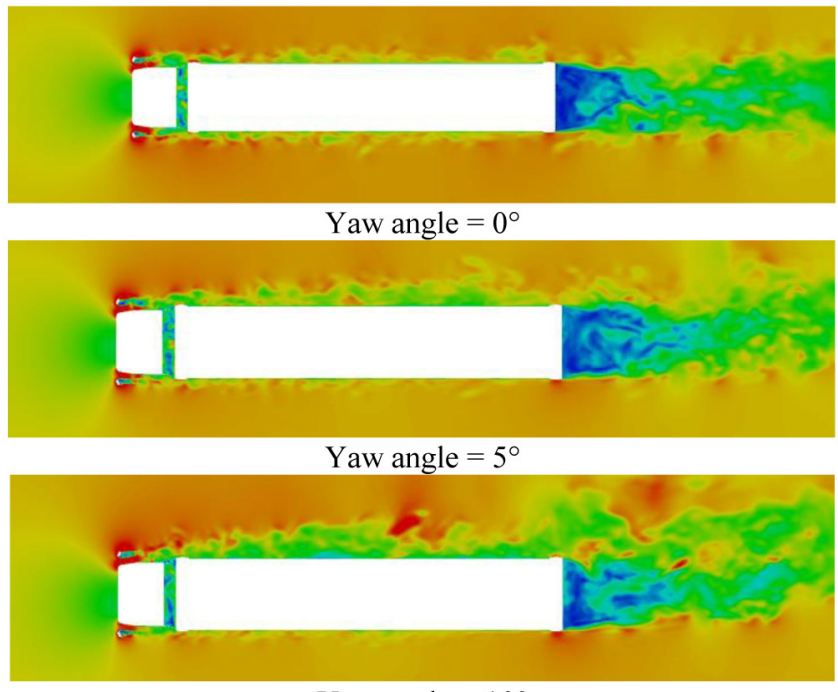

Yaw angle $=10^{\circ}$

Figure 27. Instantaneous velocity contour plot at $\mathrm{z}=2.6 \mathrm{~m}$ at different yaw angle.

The instantaneous vorticity at the constant value of 100 are visualized in Figure 28.

As discussed in the previous paragraph; the main differences between the simulations are in the regions where the devices are installed. At the yaw angle of 10 degrees; a main vorticity region is developed on the top-right part of the trailer in both the simulation suggesting the beginning of a main separation region in the right-side of the trailer. This vorticity region is responsible for the consistent increase of $\mathrm{C}_{\mathrm{Z}}$ previously shown in Table 10, 11 and Figure 26.
Target vehicle

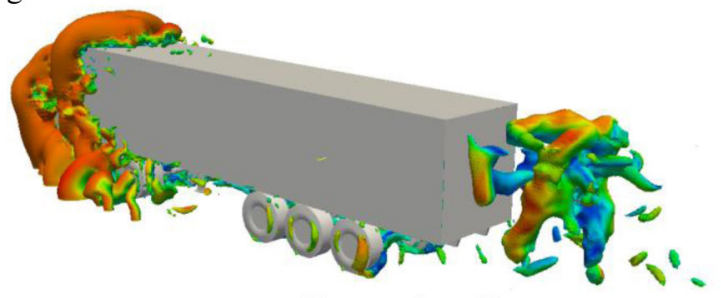

Yaw angle $=0^{\circ}$

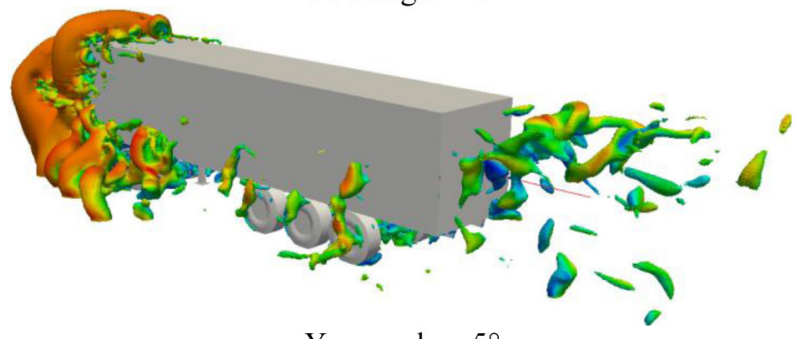

Yaw angle $=5^{\circ}$

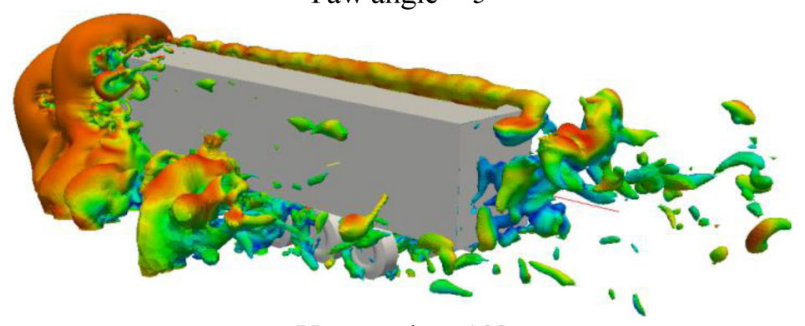

Yaw angle $=10^{\circ}$

Front-rear trailer device

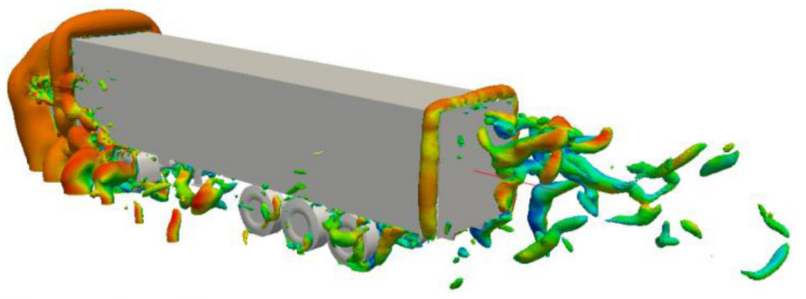

Yaw angle $=0^{\circ}$

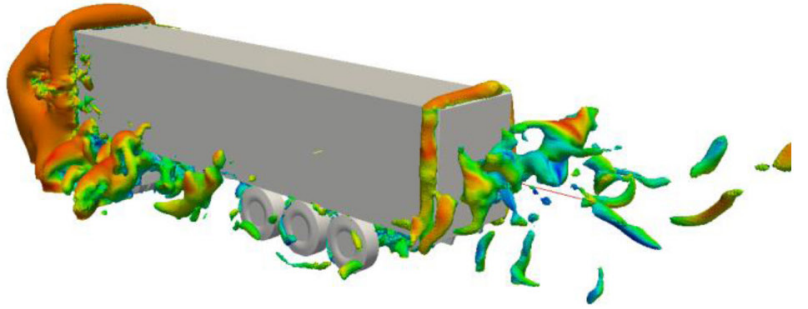

Yaw angle $=5^{\circ}$

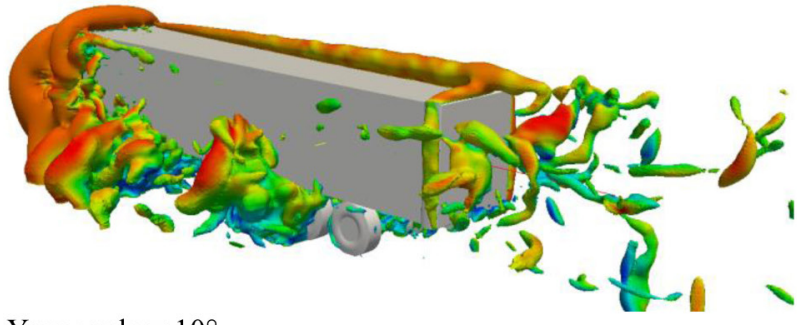

Yaw angle $=10^{\circ}$

Figure 28. Instantaneous vorticity at different yaw angle. 


\section{CONCLUSION}

A numerical model to assess the aerodynamics features of a heavy truck has been implemented in the open-source framework OpenFOAM.

This work demonstrates that installing devices with simple geometry is it possible to decrease the overall drag of the vehicle up to $12 \%$ corresponding to a fuel saving of $4 \%$ when no crosswind condition is present. Different device geometries have been tested.

The airbag solution shows capacity in reducing the drag in front wind but more studies are required to develop a shape that can have good performance also in crosswind. The possibility to use the device as an airbag in case of crash when another vehicle is approaching one with this device installed in the rear part of it, is considered a crucial point for the development of this device.

The fin can strongly decrease the drag of the vehicle although, its complex shape, makes it uncompetitive from an economical point of view.

The boat-tails are a reasonable way for reducing drag though they has safety issues for other vehicles in case of accident when this device is installed.

The front-rear trailer device produces a positive effect on the heavy truck aerodynamics both in crosswind and front wind. This device can be installed on every truck (next, current, previous generation - container) of any brand without any influence on the functionality of the vehicle. It doesn't impact to the logistic center and the shipping system: it can be easily installed/uninstalled. The access to the merchandise is not limited as happens for the airbag, fin and boat-tail solution.

This device is suitable to be used and strongly impact the transportation system with high benefit in terms of fuel efficiency and emissions into the environment.

Compared to the boat-tail, they do not raise any issue on road safety in case of crash when another vehicle is approaching one with the boat tail install in the rear part of it.

\section{ACKNOWLEDGMENTS}

We acknowledge the CINECA award under the LISA initiative, for the availability of high performance computing resources and support"

\section{REFERENCES}

1. Schoon, R., "On-Road Evaluation of Devices to Reduce Heavy Truck Aerodynamic Drag," SAE Technical Paper 2007-01-4294, 2007, doi: $10.4271 / 2007-01-4294$.

2. Patten J., McAuliffe B., Mayda W., Tanguay B. "Review of Aerodynamic Drag Reduction Devices for Heavy Trucks and Buses," Technical report National Research Council of Canada, 2012, CSTT HVC_TR-205.

3. The Council of The European Union, Council Directive 96/53/EC, July 1996.
4. Palowski F., "Wind resistance of automobiles," SAE Journal, vol. 27, pp. 5-14, 1930.

5. Hucho, W.H., “Aerodynamic of Road Vehicles,” Butterworth (Ed.), London, 1987, ISBN: 0408014229.

6. Buchheim, R., Deutenbach, K., and Lückoff, H., "Necessity and Premises for Reducing the Aerodynamic Drag of Future Passenger Cars," SAE Technical Paper 810185, 1981, doi:10.4271/810185.

7. Howell J., "The influence of ground simulation on the aerodynamics of simple car shapes with an underfloor diffuser," presented at the RaeS Conference on Vehicle Aerodynamics, Loughborough, July 1994, pp. 36.1-11.

8. Gilhaus A., "The influence of cab shape on air drag of trucks," presented at the $4^{\text {th }}$ Colloquium on Industrial Aerodynamics, Aachen, 1-20 June 1980, pp. 133-56.

9. Drollinger, R., "Heavy Duty Truck Aerodynamics," SAE Technical Paper 870001, 1987, doi:10.4271/870001.

10. Cooper, K., "Truck Aerodynamics Reborn - Lessons from the Past," SAE Technical Paper 2003-01-3376, 2003, doi:10.4271/2003-01-3376.

11. Leuschen, J., Cooper, K.R., "Summary of full-scale wind tunnel tests of aerodynamic drag-reducing devices for tractor-trailers. The Aerodynamics of Heavy Vehicles II: Trucks, Buses, and Trains," Springer, New York, 2009, pp. 451-462, doi:10.1007/978-3-540-85070$\underline{041}$.

12. Garry, K.P., "Development of container-mounted devices for reducing the aerodynamic drag of commercial vehicles," Journal of Wind Engineering and Industrial Aerodynamics 9, 113-124, 1981, doi:10.1016/0167-6105(81)90082-9.

13. Watkins, S., Saunders, J.W., Hoffman, P.H., "Comparison of road and wind-tunnel drag reductions for commercial vehicles," Journal of Wind Engineering and Industrial Aerodynamics 49, 1993, pp. 411-420.

14. Garry, K.P., "A review of commercial vehicle aerodynamic drag reduction techniques," Proceedings of the Institution of Mechanical Engineers D: Transport Engineering 199, 215-220, 1985, doi:10.1243/ PIME_PROC_1985_199_159_01.

15. Ingram K., "The wind-averaged drag coefficient applied to heavy goods vehicles," Transport and Road Research Laboratory, TRRL Supplementary Report 392, 1978.

16. McCallen, R., Salari, Ortega, J., Castellucci, P., Browand, F., Hammache, M. et al., "DOE's effort to reduce truck aerodynamic drag - joint experiments and computations lead to smart design," presented at the 34th AIAA Fluid Dynamics Conference and Exhibit, AIAA 20042249, Portland, June 22, 2004, UCRL-CONF-204819.

17. Schito, P. and braghin, F., "Numerical and Experimental Investigation on Vehicles in Platoon," SAE Int. J. Commer. Veh. 5(1):63-71, 2012, doi: $10.4271 / 2012-01-0175$.

18. SAE International Surface Vehicle Recommended Practice, "Guidelines for Aerodynamic Assessment of Medium and Heavy Commercial Ground Vehicles Using Computational Fluid Dynamics," SAE Standard J2966, Rev. Sept. 2013.

19. OpenFOAM Version 2.2.1, http:www.openfoam.org, accessed 2013.

20. Versteeg, H. K., Malalasekera W., "An Introduction to Computational Fluid Dynamics: The Finite Volume Method," 2nd edition, 2007, Pearson Education (Ed.), ISBN: 9780131274983.

21. Croll, R., Gutierrez, W., Hassan, B., Suazo, J. et al., "Experimental Investigation of the Ground Transportation Systems (GTS) Project for Heavy Vehicle Drag Reduction," SAE Technical Paper 960907, 1996, doi:10.4271/960907.

22. Salari, K. Ortega, J. "Aerodynamic Design Criteria for Class 8 Heavy Vehicles Trailer Base Devices to Attain Optimum Performance," Technical report Lawrence Livermore National Laboratory, 2010, LLNL-TR-464265.

23. Browand, F., Radovich, C., and Boivin, M., "Fuel Savings by Means of Flaps Attached to the Base of a Trailer: Field Test Results," SAE Technical Paper 2005-01-1016, 2005, doi:10.4271/2005-01-1016.

24. Salati, L., Schito, P., Cheli, F., "Dispositivo aerodinamico per la riduzione del drag di veicoli terrestri” Italian Patent MI2014A002044 November 27, 2014.

25. Das, P., Tsubokura, M., Matsuuki, T., Oshima, N., Kitoh, K. "Large Eddy simulation of the flow-field around a full-scale heavy-duty truck," presented at the 5th BSME International Conference on Thermal Engineering. 2013, pp. 521-530, doi:10.1016/j.proeng.2013.03.155.

26. Barnard, R. H., "Road Vehicle aerodynamics design: An introduction", 3rd edition, 2009, MechAero Publishing (Ed.), ISBN 0-9840734-7-3. 
27. Zulfaa, M. K., Filippone A. "Fuel savings on a heavy vehicle via aerodynamic drag reduction," Transportation Research Part D:

Transport and Environment. Volume 15, Issue5, July 2010, p.275-284, doi:10.1016/j.trd.2010.02.010.

28. Clark, N., Daley, J., Nine, R., and Atkinson, C., "Application of the New City-Suburban Heavy Vehicle Route (CSHVR) to Truck Emissions Characterization," SAE Technical Paper 1999-01-1467, 1999, doi:10.4271/1999-01-1467.

29. Cooper, K., "The Effect of Front-Edge Rounding and Rear-Edge Shaping on the Aerodynamic Drag of Bluff Vehicles in Ground Proximity," SAE Technical Paper $\underline{850288}$, 1985, doi: $10.4271 / 850288$.

30. Hucho, W.-H., "Performance of Cars and Light Trucks", "Aerodynamics of Road Vehicles, Fourth Edition," (Warrendale, SAE, International, 1998), ISBN 978-0-7680-0029-0.

31. Englar, R., "Advanced Aerodynamic Devices to Improve the

Performance, Economics, Handling and Safety of Heavy Vehicles," SAE Technical Paper 2001-01-2072, 2001, doi:10.4271/2001-01-2072.

32. Gillespie, T., "Fundamentals of Vehicle Dynamics," (Warrendale, Society of Automotive Engineers, Inc., 1992), doi:10.4271/R114.

33. Hucho, W.-H., “Aerodynamics of Road Vehicles," (Warrendale, SAE International, 1998), ISBN 978-0-7680-0029-0.

34. Transport Statistics Publications, "Transport Statistics Great Britain, 2008 ed.," Department for Transport, London, 2008, ISBN: 978-0-11553030-2.

35. Wood, R., "Impact of Advanced Aerodynamic Technology on Transportation Energy Consumption," SAE Technical Paper 2004-01$\underline{1306}$, 2004, doi:10.4271/2004-01-1306. 